\title{
Pediatric Knowledge About Acute Viral Hepatitis
}

\author{
Rita Franca ${ }^{1}$, Luciana Silva ${ }^{2}$, Maria Clotildes Melo, \\ Suzy Cavalcante ${ }^{1}$, Bruno Lima $^{3}$,Anita Rocha ${ }^{3}$, \\ Cristiana Gomes ${ }^{3}$ and Mônica Franca ${ }^{4}$
}

\author{
Gastroenterology and Pediatric Hepatology Division ${ }^{1}$, \\ Pediatric Department ${ }^{2}$, School of Medicine, School of \\ Medicine ${ }^{3}$, School of Odontology ${ }^{4}$, Federal University \\ of Bahia, Salvador, BA, Brazil
}

\begin{abstract}
Knowledge about hepatotropic viruses is crucial for pediatricians because of the high prevalence of viral hepatitis during childhood. The multiplicity of hepatotropic viruses, the spectrum of acute and chronic infections, and the sequels of viral hepatitis result in a need for physicians to better understand the clinical and epidemiological context of patients with viral hepatitis, as well as the importance of prevention measures for hepatitis. A descriptive cross-sectional study was made of pediatrician's knowledge about viral hepatitis, through questionnaires to 574 pediatricians, with no obligation of identification. The pediatricians were recruited among those who attended a national Congress of Pediatrics in Brasília, Brazil. Among these pediatricians, $50.1 \%$ frequently treated cases of hepatitis, and $74.7 \%$ indicated that they had knowledge of the existence of five hepatotropic viruses; $14.5 \%$ knew about at least four types of hepatitis complications, while only 7.7\% and $4.3 \%$ were able to correctly diagnose viral hepatitis $A$ and $B$, respectively. Many $\mathbf{2 8 . 4 \%}$ ) did not know how to treat the patients adequately. Only $37.5 \%$ had already recommended vaccination against hepatitis B. Only $50.2 \%$ of the pediatricians had been vaccinated against hepatitis $B$. We concluded that it is crucial to make pediatricians more knowledgeable about viral hepatitis, through continued education programs, especially emphasizing prevention procedures. Key Words: Hepatitis, pediatrics.
\end{abstract}

Hepatitis is a diffuse inflammatory process of the liver, with various causes, such as hepatotropic and non-hepatotropic viruses, drugs, metabolic diseases (Wilson's disease, alpha 1 -antitrypsin deficiency) and autoimmune phenomena. Viral infections are the most important causes of acute hepatic diseases in the world. They consist of systemic infections in which the predominant manifestations are due to hepatic lesion and dysfunction. The term viral hepatitis generally refers to hepatic lesion caused by hepatotropic viruses. Currently known types are A, B, C, D, and E; G virus and TT virus do not cause liver disease [1,2]. Hepatitis viruses cause a diversity of clinical features, which range

Received on 30 November 2003; revised 11 May 2004.

Address for correspondence: Dr. Rita de Cássia Franca. Rua Macapá, 461, apt.701, Ondina. Zip code: 40170-150, Salvador, BA, Brazil.Phone/Fax:(5571)237-1472.E-mail: rcfmelo03@ig.com.br

The Brazilian Journal of Infectious Diseases 2004;8(3):227-235 (C) 2004 by The Brazilian Journal of Infectious Diseases and Contexto Publishing. All rights reserved. from a state of asymptomatic infection to acute or fulminate hepatitis; some of the hepatotropic viruses (B, C, and D) may evolve to chronic disease, hepatic cirrhosis, or hepatocellular carcinoma.

Acute viral hepatitis is an important cause of child morbidity. Chronic hepatitis is also relevant in the pediatric age range in relation to morbidity and mortality in infants. Non-hepatotropic viruses such as mumps, mononucleosis, cytomegalovirus, herpes virus, varicella, rubella, measles, and others can also cause hepatitis. Nowadays, acute viral hepatitis treatment is supportive: good hydration, symptom relief, and bed rest, according to the patient's state of health.

The World Health Organization (WHO) reports that more than 2 billion individuals have had contact with the hepatitis B virus, being responsible for 1 to 2 million deaths/year throughout the world. It is estimated that the number of chronic carriers may be as high as 200300 million and that $25 \%$ of them run a great risk of suffering serious affections and of dying of hepatic 
cirrhosis or liver cancer. Almost all of those problems would be prevented if hepatitis B vaccines, available since 1982 [3], were more widely used. Hepatitis B vaccine was previously recommended only to high-risk groups. However, due to the poor results in lowering the number of carriers with this selective immunization [4], several organizations, such as The American Academy of Pediatrics, The American Academy of Family Physicians, the Advisory Committee of Immunization Practice, and the WHO have recommended the expansion of immunization coverage, with mandatory hepatitis B universal immunization of nursing infants [3-5].

The first hepatitis B vaccines were known as plasmaderived vaccines. They consisted of purified hepatitis B surface antigen, AgHBs, taken from the blood of chronic carriers. However, as the production cycle took longer than a year, the supplies of such vaccines were rather limited. Progress in molecular biology has permitted the development of sophisticated and highly reliable genetic engineering techniques. This has led to the introduction of vaccines produced by genetic engineering; these are produced in mammalian cells or in yeast; none of them includes blood in their preparation. The first recombinant vaccine, Recombix, produced by Merck Sharp and Dohme, was licensed in the United States in 1986, followed by another of similar composition, Engerix B, produced by Smith Kline Beecham, in 1989 [3,6]. Based on clinical studies, the vaccine is known to provide immunization in over $96 \%$ of the recipients, one month after the third dose [7]. This vaccine induces active synthesis of anti-HBs, together with an immunological memory for $\mathrm{AgHBs}$, independent of the quantity of antibodies. Complementary studies have shown that the number of memory lymphocyte $B$ cells, capable of producing anti-HBs, does not diminish with decreases in antibody levels.

Serologic studies have demonstrated immunologic memory, which is produced by effective immunization for periods of over 5 years in recipients who are often exposed to hepatitis B virus. Thus, immunization reinforcement seems unnecessary in healthy individuals when anti-HBs levels are below $10 \mathrm{mIU} / \mathrm{mL}$. Recent research has suggested that good immunologic memory in healthy immunized persons lasts more than 5-12 years. The most frequently used immunization schedule consists of 3 intramuscular doses at 0,1 and 6 months [3]. Adverse effects are generally weak and are restricted to the first day after administration. Topic reactions are more common and consist of transient pain, erythema, and stiffening. The early systemic events include pruritis, angioedema, urticaria, and bronchospasm-like symptoms. Anaphylaxis is extremely rare. The reported systemic adverse effects chronologically associated with immunization encompass fatigue, dizziness, syncope, hypotension, arthritis, arthralgia, lymphadenopathy, urticaria, and rash. Flu-like symptoms (low fever, malaise, headaches, and myalgia) or gastrointestinal ones (abdominal pain, diarrhea, vomiting, nausea, and serologic hepatic changes) have rarely been described. Neurological manifestations, such as paralysis, paresthesia, neuropathy, neuritis, including Guillian-Barré syndrome, optical neuritis, and multiple sclerosis, have rarely been described. Major skin disorders, like multiform erythema, have been extremely rare. Adverse effects described in newly born and nursing infants include fever, agitation, and apnea [5].

Success achieved with hepatitis B vaccine has led to hepatitis A vaccine. The first active vaccine against hepatitis A virus was introduced in 1992; it has several advantages when compared to immunoglobulin. It is well tolerated, effective, and results in persistent high rates of seropositivity. Recent research has described the vaccine produced by Merck Sharp and Dohme and SmithKline Beecham. Immunization causes seroconversion in approximately $100 \%$ of recipients $[6,8]$. The inactivated vaccine is administered intramuscularly following the 0,1 and 6-month schedule [9]. Hepatitis A vaccine has been reported as safe, well tolerated, and highly immunogenic to both adults and children in over 104 studies, which have been carried out in 27 countries with approximately 50,000 people and 120,000 vaccine doses. The recommendation for groups to be immunized remains, nonetheless, an open question. The high costs, as well as epidemiological considerations, based on studies carried out in countries with greater availability of hepatitis A vaccine have 
resulted in its not yet being recommended its use on a large scale. However, some authors think that without universal immunization, a real decrease in hepatitis A virus transmission is unlikely. The recommendation of vaccines for travelers going to places where hepatitis A is endemic is consensual.

In developed countries, immunization of groups analogous to the ones which have received hepatitis B vaccination has been proposed; these include institutionalized individuals, health care workers, catering workers, people with multiple sexual partners or hepatitis B carrier partners, intravenous drug users, chronic hepatic patients, and hemophilia patients [10,11].

Inactivated anti-hepatitis A vaccines is well tolerated; this tolerance increases with the number of doses, highlighting the absence of sensitivity. Children have presented fewer post-vaccination reactions than adults. Topic reactions, provoked by the adjuvant, have been restricted to pain at the spot where the injection was administrated, followed by erythema and local edema. The reported systemic adverse reactions have been essentially weak, lasting no longer than 3 days; they include headaches, malaise, fatigue, fever, nausea, anorexia, and arthralgia. All of these have spontaneously disappeared. Elevation of transaminase is rare [11-14]).

Great advances in the field of viral hepatitis have resulted in a need for doctors to better understand the epidemiological and clinical contexts of patients with viral hepatitis, as well as the importance of pediatric practice in preventing these diseases.

We sought to identify the pediatricians' knowledge of acute viral hepatitis, including the types of hepatotropic viruses, diagnosis, prophylaxis of acute hepatitis A and B, and patient care. Other objectives were to assess post-graduate qualification among pediatricians and to determine the percentage professionals vaccinated against hepatitis B.

\section{Materials and Methods}

We made a descriptive cross-sectional study, in which pediatric practice and knowledge of hepatitis were evaluated. A total of 574 pediatricians out of the 2,000 who attended a Pediatric Course in Brasilia, Brazil, took part in the study. Pediatricians from all over the country are usually present at this annual course. Information was obtained by having the professionals answer a questionnaire. Each individual had at least one year's practice, although no specialization certificate was required. Those who refused to answer the questionnaire, and undergraduates, were excluded.

The questionnaire consisted of items about postgraduate qualification among pediatricians, that is, a master's degree and residency in Medicine, questions to assess their knowledge of acute viral hepatitis etiology (which viruses are hepatotropic and their complications), the frequency of cases of hepatitis (how they use laboratory tests in order to identify hepatitis A and hepatitis B), and also questions related to treatment and prophylaxis (therapeutic management of acute viral hepatitis, routine immunization schedule, knowledge of vaccines against hepatitis). The professionals were also asked whether they had been vaccinated against hepatitis B.

The variables were analyzed as follows:

- Post-graduate qualification - only yes and no answers were quantified.

- Pediatricians' routine immunization orientation there were three categories, with quantified results: the number of professionals who just responded to the simple vaccination schedule (polio vaccine, DTP, $\mathrm{BCG}$, and vaccine against measles); the ones who added other vaccines to the routine schedule, and also the ones who recommended other vaccines, such as HAV vaccine, HBV vaccine, MMR vaccine, and anti-Haemophilus influenzae vaccine.

- Frequency of managed hepatitis B cases - this item totaled the numbers of yes and no answers.

- Knowledge of hepatotropic viral hepatitis - in this item 3 categories were considered: "know about it" (when 5 of the types of hepatitis viruses: A, B, 
C, D, and E were correctly mentioned); "Know partially about it" (when at least 3 of those types were answered); and "do not know about it" (when fewer than 3 types were mentioned, or the answers were incorrect).

- Knowledge of how to diagnose acute viral hepatitis A and hepatitis B through the interpretation of laboratory tests - three categories were analyzed in this item: "know about it" (when high levels of transaminase and anti-HAV IgM for hepatitis A and high levels of transaminase, AgHBs, and antiHbcIgM for hepatitis B were the answers); "Know partially about it" (for both hepatitis A and hepatitis $\mathrm{B}$, when at least one of the answers was correct); and "do not know about it" (when the answers were incorrect).

- Knowledge of management of acute viral hepatitis patient care - the following criteria were considered: diet, hydration, personal hygiene, attention to encephalopathy signs, avoidance of hepatotoxic drugs, and increased bed rest. The categories were established based on the answers: "know about it" (when 3 or more criteria were mentioned), "Know partially about it" (when fewer than 3 criteria were mentioned), and "do not know about it" (when incorrect answers were given).

- Knowledge of which types of hepatitis vaccine exist - The categories were established based on the answers: "know about it" (when answered for hepatitis A and hepatitis B), "Know partially about it" (when just one or the other was answered), and "do not know about it" (when incorrect answers were given).

- Immunization recommendation - the numbers of yes and no answers were totaled.

- Knowledge of immunization effectiveness - the following criteria were considered: coverage period over 5 years, effectiveness percentage around 90$95 \%$, capability of protecting against major types and/or a state of chronicity, and high effectiveness.
The three categories were established based on the answers: "know about it" (when 3 or more criteria above were mentioned), "Know partially about it" (when fewer than 3 of them were mentioned), and "do not know about it" (when incorrect answers were given).

- Knowledge of hepatitis vaccine side effects - - two kinds of effects were considered: local side effects (pain, heat, reddening of the area, and edema) and systemic side effects (fever, headache, nausea, dizziness, fatigue, and neurological disorders). The three categories were established based on the answers: "know about it" (when at least 3 effects were mentioned), "Know partially about it" (when fewer than 3 of them were mentioned), and "do not know about it" (when incorrect answers were given).

- Knowledge of the possible hepatitis complications: the following complications were considered: cirrhosis, hepatocarcinoma, fulminant hepatitis, hepatic failure, chronic hepatitis, glomerulonephritis, and aplastic anemia. The analyzed categories were: "know about it" (when 4 of these complications were correctly mentioned); "Know partially about it" (when fewer than 4 were correctly answered); and "do not know about it" (when incorrect answers were given).

- If the professional was vaccinated against hepatitis $\mathrm{B}$ - the numbers of yes and no answers were totaled.

\section{Results}

Out of 574 pediatricians, $391(68.1 \%)$ had medical residency in Pediatrics, 158 (27.5\%) did not have it, and $25(4.4 \%)$ did not answer this question. On the other hand, only 8 pediatricians $(1.5 \%)$ had a Master's degree, $430(74.9 \%)$ answered that they did not have such a degree, and 136 (23.6\%) did not answer.

For the questions about types of hepatitis caused by known hepatotropic viruses, 429 pediatricians $(74.7 \%)$ answered correctly about the 5 types of hepatitis (A, B, 
C, D, and E), 122 (21.3\%) were able to answer partially, about at least 3 types, $18(3.1 \%)$ were unable to answer correctly, and 5 (0.9\%) did not answer.

As seen in Figure 1, only $83(14.5 \%)$ had knowledge of at least 4 of the complications, 427 (74.7\%) had partial knowledge of the possible complications, $26(4.5 \%)$ did not have such knowledge, and 38 (6.6\%) did not answer.

Concerning the frequency of managed cases, 339 (59.1\%) pediatricians often saw this kind of pathology, $227(39.5 \%)$ do not, and $8(1.4 \%)$ did not answer.

Figures 2 and 3 refer to the knowledge of all pediatricians regarding how to diagnose acute viral hepatitis A and hepatitis B through the interpretation of laboratory tests. Only 44 (7.7\%) were able to correctly interpret the laboratory tests on hepatitis A. The incomplete answers (high level of transaminase and antiHAV) were also considered correct. Just 25 (4.3\%) of the pediatricians gave a completely correct answer; 361 professionals $(62.9 \%)$ were able to diagnose partially, $127(22.1 \%)$ were not able to answer correctly, and 42 (7.3\%) did not answer. Only $23(4 \%)$ pediatricians were able to correctly diagnose hepatitis B. Just 7 of them gave a complete answer (antiHbcIgM); 395 (68.8\%) were able to answer partially, $115(20 \%)$ were unable to give correct answers, and $41(7.2 \%)$ did not answer.

In the investigation of knowledge of management of acute viral hepatitis patient care, out of 574 professionals, 121 (21.1\%) knew how to handle their patients correctly, answering 3 or more of the adopted criteria, 266 pediatricians knew partially how to do it, $163(28.4 \%)$ were considered unable to handle the patients correctly, and 14 (24\%) did not answer (Figure 4). Eighty (14\%) professionals made use of outdated guidelines, such as the use of hepatoprotectors and diets rich in sugar and low in fat. Those who made use of outdated guidelines concerning diet alone, but got the three other criteria right, were included in the "Knowpartially-about-it" group. However, when professionals added the use of medications such as hepatoprotectors, B complex vitamins, among others, they were included in the "do-not-know-about-it" group, regardless of other correct answers.
In answer to the questions about routine immunization orientation, $238(41.5 \%)$ professionals only followed the WHO basic schedule, $328(57.1 \%)$ also recommended other vaccines, such as MMR vaccine, $\mathrm{HBV}$ vaccine, and anti-Haemophilus influenzae, and $8(1.4 \%)$ did not answer. Concerning the knowledge of which types of hepatitis vaccine exist, $220(38.3 \%)$ pediatricians knew about the existence of vaccines against hepatitis $\mathrm{A}$ and hepatitis B, 338 (58.9\%) only knew about vaccines against hepatitis B, 7 (1.2\%) declared that they did not know about the existence of such a vaccine, and 9 (1.6\%) did not answer. Only 215 pediatricians (37.5\%) had already recommended immunization against hepatitis, whereas $353(61.5 \%)$ had never done so, and $6(1 \%)$ did not answer the question (Figure 5).

Just 288 pediatricians $(50.2 \%)$ had already been vaccinated against hepatitis B, 257 (44.8\%) had not yet been vaccinated, and $29(5.0 \%)$ did not answer the question. Regarding knowledge of immunization effectiveness, it was observed that only 2 professionals $(0.3 \%)$ knew the answer; 447 (77.9\%) demonstrated partial knowledge of it, and 44 (7.7\%) did not answer. Concerning vaccine side effects, only 33 professionals (5.7\%) knew about them, 126 (22\%) knew partially about these effects, $334(58.2 \%)$ could not answer, and $81(14.1 \%)$ did not answer.

\section{Discussion}

Knowledge of hepatitis among pediatricians' who attended the congress can be regarded as deficient. Out of the 574 professionals who answered the questionnaire, $68.1 \%$ had medical residency in Pediatrics, and only $1.5 \%$ had a master's degree. Apparently not all of these professionals had access to specialization or to updated information.

It is necessary for medical schools to provide real learning conditions to help health care professionals, so that they can perform their roles competently. It is also important for all doctors, even the newly graduated, to have access to the Residency in Medicine, or a similar system, in order to assure proper training and competence. 


\section{Figure 1.}

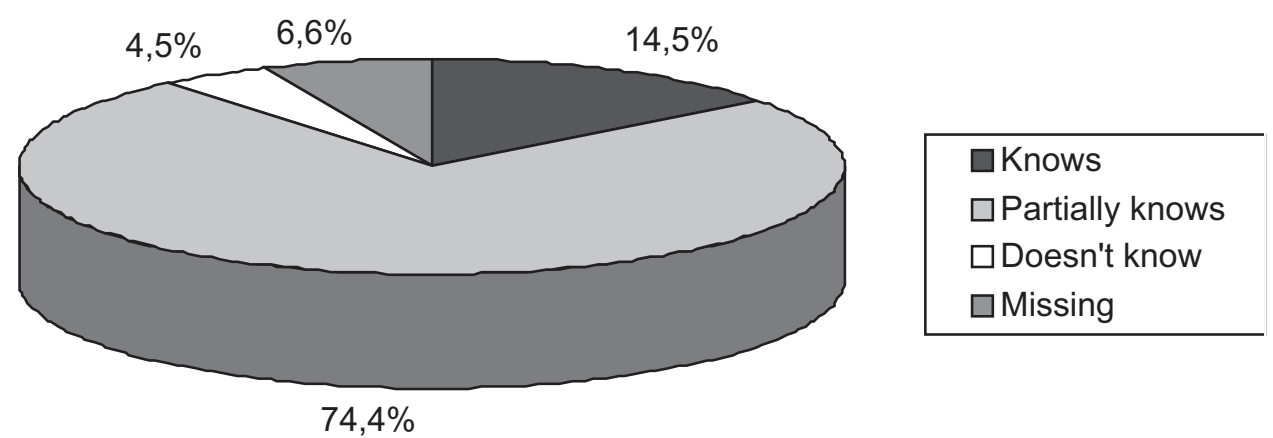

Graphic 1: Knowledge of Pediatricians about the Complications of Hepatitis

\section{Figure 2.}

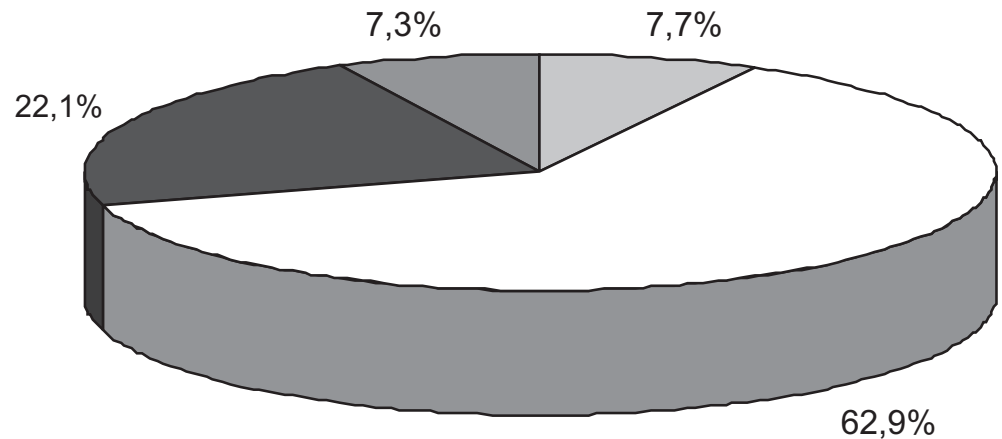

$\square$ Knows

$\square$ Partially knows

口Doesn't know

$\square$ Missing

Graphic 2.Knowledge of Pediatricians about the Laboratory Diagnosis of Acute Hepatitis A 
Figure 3.

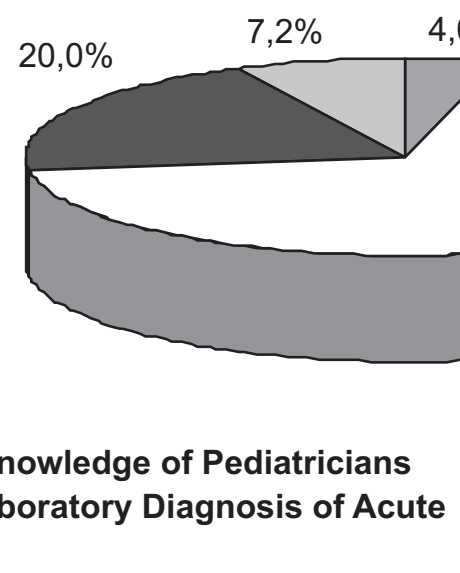

Graphic 3: Knowledge of Pediatricians about the Laboratory Diagnosis of Acute Hepatitis B

\section{Figure 4.}

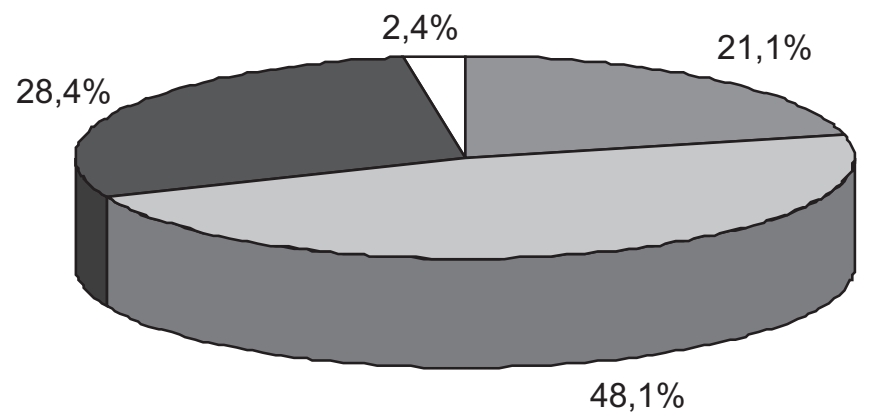

Knows

$\square$ Partially knows

$\square$ Doesn't know

$\square$ Missing

Graphic 4: Knowledge of Pediatricians about the Treatment of Viral Acute Hepatitis 


\section{Figure 5.}
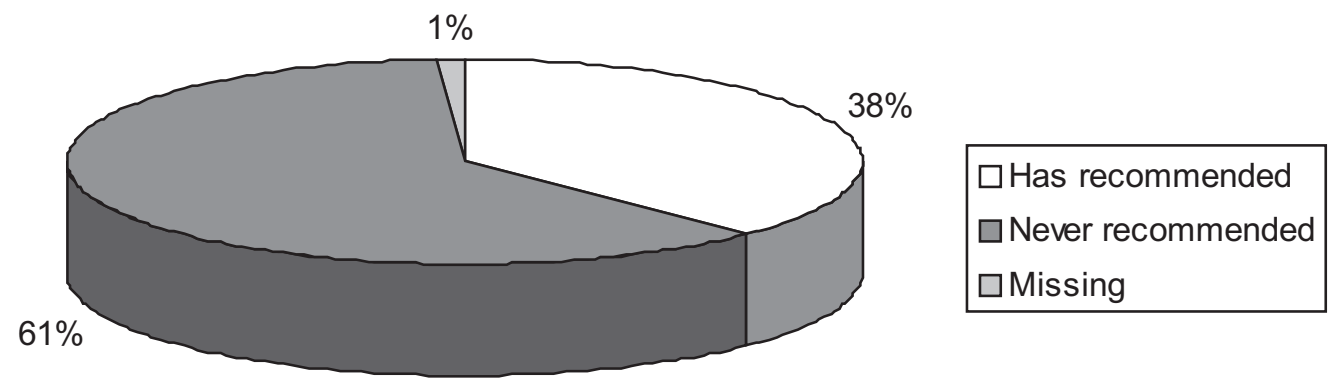

\section{Graphic 5: Orientation of Pediatricians towards Hepatitis B Vaccination}

Medical schools have been trying to bridge this gap in their courses, by re-evaluating and changing their curricula so as to focus the practice on one of the 4 major areas (Pediatrics, Surgery, Clinical Medicine, or Gynecology and Obstetrics) during the last year of their curriculum. This is a good starting point, which will certainly improve the quality of medical teaching, but it does not fulfill the need for extending knowledge and periodically refreshing it with the creation of further education programs.

The number of viral hepatitis diagnostic methods has recently increased, beyond the simple screening for transaminase. Thus, for acute hepatitis A diagnosis, the most commonly used method is anti-HAV IgM; anti-HBc IgM for acute hepatitis B, and anti-HEV IgM for acute hepatitis E. Anti-HCV and PCR markers are used to detect infection by the $\mathrm{C}$ virus. Anti-HDV and $\mathrm{IgM}$ and $\mathrm{IgG}$ markers are used to detect infection caused by the D virus; anti-HGV and PCR detect the $\mathrm{G}$ virus. There are a great number of methods to diagnose viral hepatitis. However, when the pediatricians' knowledge of types of hepatitis viruses was analyzed, $74.4 \%$ knew of the existence of 5 types of viruses (A, B, C, D and E), but only $7.7 \%$ and $4.3 \%$ knew how to correctly diagnose acute viral hepatitis A and B through laboratory tests, respectively. These figures clearly indicate a lack of knowledge.
The most frequent complications derived from hepatitis B, C, and D, are a carrier state, followed by chronic hepatitis, cirrhosis, hepatocarcinoma, fulminant hepatitis and aplastic anemia. In cases of hepatitis $\mathrm{A}$ and hepatitis $\mathrm{E}$, the most important complication is fulminant hepatitis, since these types of hepatitis do not evolve to chronicity. Only $14.5 \%$ of the pediatricians correctly knew at least 4 types of possible hepatic complications. These figures show these professionals' poor knowledge of possible complications and lab methods used to diagnose correctly the hepatitis.

Some patients may not be correctly diagnosed, and many opportunities to adequately immunize the individuals may be missed. On the other hand, it was observed that approximately $60 \%$ of these professionals frequently handled hepatitis cases.

The hepatitis regimen has been based on empirical measures for a long time. Based on some studies, it was found that treatment should only involve dealing with symptoms and hydration. In analyzing the data on the pediatricians' knowledge of how to manage the patient with hepatitis, it was observed that the number of professionals who could not handle the patient properly was superior to the number of the ones who could do so correctly ( $28.4 \%$ and $21.1 \%$ respectively). Many (14\%) of the pediatricians made use of outdated 
guidelines, such as the use of hepatoprotectors and diets rich in sugar and low in fat.

Though the majority of the pediatricians knew that there was a vaccine against hepatitis B, only $37.5 \%$ had already recommended its use. This data is similar to the figures given by Freed et al. (1994). They found that $82 \%$ of pediatricians had knowledge of recommendations of the American Academy of Pediatrics, the American Academy of Family Physicians, and the Advisory Committee of Immunization Practice, for universal immunization of nursing children against hepatitis B, but only $37 \%$ had already followed them.

In this study, no pediatrician recommended immunization against hepatitis $\mathrm{A}$. This might be due to the fact that the vaccine had just been introduced. Only $50 \%$ of pediatricians were vaccinated against hepatitis B. Immunization is essential to health care workers, especially to those at great risk, such as surgeons, lab workers, and pediatricians.

When knowledge of immunization effectiveness and side effects of hepatitis vaccines was considered, only $0.3 \%$ and $5.7 \%$, respectively, knew something, which supports the idea that the pediatricians know very little about such vaccines, and that they know even less about its effectiveness and side effects.

We found that there is an urgent need for creating a greater stimulus to further education and easier access to this education. Such education should focus not only on the disease, but also on its treatment and prophylaxis, which should be better publicized. The recent advances in technology and knowledge of Medicine should be considered in the light of the major objective of the medical profession, which is health of the population at lower costs. It is also important that medical services provide a means of evaluating their staff performance in order to update them periodically.
2. Paraná R. Estudo Clínico Sorológico da Hepatite Aguda Não-A Não-B Esporádica em Salvador-Bahia. Tese de Doutorado. Universidade Federal da Bahia, 122, 1997.

3. Rodrigues D., Bricks F.L., Resegue R. Hepatite B: Imunização Universal. Pediatria ( São Paulo) 1996;18(2): 82-90.

4. Hall B.C., Halsey N.A. Control of Hepatitis B: To be or not to be? Pediatrics 1992;90(2):274-7.

5. West D.J., Caladra G.B. Vaccine Induced Immunologic Memory for Hepatitis B Surface Antigen: Implications for Policy on Booster Vaccination. Vaccine (England) 1996; 14 (11):1019-27.

6. Strader D.B., Seeff L.B. New Hepatitis A Vaccines and Their Role in Prevention. Drugs 1996;51(3):359-66.

7. Steffen R., Gyurech D. Advances in Hepatitis A Prevention in Travellers. Journal of Medical Virology 1994;44:4602.

8. Balli F., Di Biasi A.R., Viola L. Vaccination Against Hepatitis A. Pediatr Med Chir 1996;18(3):259-62.

9. Van Damme, Kane M., Meheus A. Integration of Hepatitis B Vaccination into National Immunisation Programmes. Viral Hepatitis Prevention Board. BMJ (England) 1997;5:1036-7.

10. Barsanti M. Hepatite A: Recomendações quanto à imunoprofilaxia. Pediatria Moderna - Guia de Atualização Terapêutica, [São Paulo],1997.

11. Freire H. Hepatite A - Profilaxia. Documentos Científicos da Sociedade Brasileira de Pediatria (SBP). Departamento de Infectologia, no. ${ }^{\circ}$ 2, 1997.

12. Balcarek B.K., Bagley R.M., Pass F.R., et al. Safety and Immunogenicity of an Inactivated Hepatitis A Vaccine in Preschool Children. The Journal of Infectious Diseases 1995;171(suppl 1): S70-2.

13. Langer A.C.B., Lövestad, Frösner G.G. High Immunogenicity and Good Tolerability of a New Hepatitis A Vaccine Candidate. Vaccine 1996;14(12):1089-91.

14. Smithkline Beechan. Monografia da Havrix: Vacina Contra Hepatite A. Copyright, 26.

\section{References}

1. Ferreira C., Silveira T. Hepatites virais: atualização. Jornal de Pediatria 1997;73(6):367-76. 